\title{
Quand le principe de précaution frappe à la porte des constitutions
}

\section{When the precautionary principle knocks at the door of constitutions}

\author{
Olivier Godard ${ }^{1}$ \\ Économiste, Laboratoire d'économétrie, UMR 7657 CNRS/École polytechnique 1 rue Descartes, \\ 75005 Paris, France
}

L'année 2003 aura été en France et en Europe l'année des refondations constitutionnelles. D'abord avec le projet de Charte de l'environnement destinée à être adossée à la Constitution française. Ensuite avec le projet de Constitution européenne. La préparation de ces deux textes a avivé de fortes tensions. Ce fut plus surprenant pour le premier que pour le second. À première vue, la Charte ne devait faire que consacrer symboliquement des valeurs et des concepts déjà bien intégrés au droit européen et national. Pas de quoi s'émouvoir puisque, du fait du traité de Maastricht, les principes pollueur-payeur et de précaution étaient déjà applicables sur le sol français depuis une décennie au moins, les engagements internationaux s'imposant aux législations nationales. Il n'en fut que plus étonnant de voir se lever un vent de réaction contre l'idée de voir ces principes inscrits dans la Charte.

C'est dans les milieux industriels et les milieux médicaux que ce vent s'est levé, bien vite relayés par l'Académie nationale de médecine le 11 mars 2003, puis par l'Académie des sciences le 18 mars 2003. Cette dernière recommandait alors « que le principe de précaution ne soit pas inscrit dans des textes à valeur constitutionnelle ou dans une loi organique car il pourrait induire des effets pervers,

Adresse e-mail : godard@poly.polytechnique.fr (0. Godard).

1 Economiste, chercheur au CNRS, Olivier Godard a été membre du Comité scientifique auprès de la Commission Coppens de préparation de la Charte de l'environnement. susceptibles d'avoir des conséquences désastreuses sur les progrès futurs de notre bien-être, de notre santé et de notre environnement ».

Pourquoi donc ces académies dont la seule légitimité est scientifique ont-elles quitté leur domaine de compétence pour intervenir directement dans le champ politique au nom du bien-être, de la santé, du développement économique et même de cet environnement jugé si menacé par une Charte qui voulait consacrer les droits et devoirs des citoyens à son endroit ? Ce sont les risques de dérive des principes de précaution et de pollueur-payeur, mais aussi de l'idée de développement durable qui servent de trame commune à l'argumentaire de ces frondeurs. Qu'en penser?

D'abord que c'est une bien étrange façon d'aborder un problème de la part de scientifiques. Lorsque des dérives existent, et ce n'est pas une hypothèse d'école, elles doivent être combattues avec vigueur dans le débat public. Mais il ne faut pas se tromper de cible. Appréhender un texte et les notions qu'il développe, non en critiquant ce qu'ils sont, mais en fonction des déformations et travestissements qu'ils pourraient subir et dont ils seraient les victimes n'est pas de bonne méthode... Faudrait-il renoncer à proclamer les valeurs de liberté et d'égalité au motif que l'une et l'autre peuvent connaître dérives et trahisons que l'expérience historique du $X X^{e}$ siècle a illustré d'abondance ? Brandir des dérives dont on dit qu'elles ont déjà eu lieu pour condamner un texte constitutionnel à venir témoigne d'un sens de la logique plutôt curieux. N'est-ce pas l'inverse qu'on doit soutenir, 
à savoir que la formulation de ces principes et valeurs, dans un texte placé sous la vigilance du Conseil constitutionnel, est le moyen de mettre un terme aux dérives craintes?

Il a fallu l'intervention et l'obstination, sur ce point, du président de la République pour que le principe de précaution soit maintenu dans le projet de Charte. C'est finalement le seul principe mentionné comme tel, à l'article 5 , de la façon suivante: «Lorsque la réalisation d'un dommage, bien qu'incertaine en l'état des connaissances scientifiques, pourrait affecter de manière grave et irréversible l'environnement, les autorités publiques veillent, par application du principe de précaution, à l'adoption de mesures provisoires et proportionnées afin d'éviter la réalisation du dommage ainsi qu'à la mise en œuvre de procédures d'évaluation des risques encourus ». Cette formule pourrait sans doute être encore améliorée de plusieurs manières. Il aurait été préférable de s'en tenir à la formulation d'origine de la Déclaration de Rio qui se référait à des dommages graves ou irréversibles pour qualifier les circonstances d'enclenchement de procédures d'évaluation et de prévention de ces risques. Il aurait également été préférable de demander des mesures révisables, plutôt que provisoires. Songeons aux politiques de l'effet de serre qui vont vraisemblablement jalonner l'histoire de l'humanité tout au long du XXI ${ }^{\mathrm{e}}$ siècle ! Surtout, le but fixé à l'action de précaution, « éviter la réalisation du dommage », est ambigu. La formule pourrait laisser croire qu'il est possible et souhaitable de viser en toutes circonstances l'éradication des risques, si la référence à la proportionnalité ne venait faire contrepoids. Un but plus ouvert, comme « éliminer ou réduire le danger et diminuer l'ampleur du dommage possible ", aurait mieux convenu.

En dépit de ces réserves, ce texte a l'avantage d'affirmer sans ambages qu'il n'y a pas lieu d'attendre des certitudes scientifiques pour engager une prévention et que le devoir de première initiative incombe aux autorités publiques, s'agissant d'environnement, dont la Charte rappelle qu'il est un patrimoine commun des êtres humains. En obligeant les autorités à mettre sur pied une évaluation des risques, il écarte toute velléité d'opposer le principe de précaution et l'expertise scientifique. Allant dans le même sens, l'article 9 de la Charte proclame que « la recherche et l'innovation doivent apporter leur concours à la préservation et à la mise en valeur de l'environnement ». Pas question, ici, de cultiver l'opposition entre protection de l'environnement et recherche, ni de s'en tenir au seul volet « protection ». Cependant, en posant une obligation, cet énoncé manifeste aussi que cela ne va pas de soi ; un effort spécifique, une organisation, seront nécessaires pour que recherche et innovation apportent le concours demandé.

Attention, un train peut en cacher un autre ! Charte de l'environnement ou pas, les Français sont confrontés au projet de Constitution de l'Union européenne. Préparé sous la férule de Valéry Giscard d'Estaing, ce projet fixe notamment à l'Union l'objectif d'œuvrer pour le développement durable de l'Europe. Son article III-129 affirme aussi que la politique de l'Union est fondée sur les principes de précaution et d'action préventive, sur le principe de la correction, par priorité à la source, des atteintes à l'environnement et sur le principe du pollueur-payeur. Ces formulations, qui étaient déjà celles du traité de Maastricht, se trouvent ici portées au niveau constitutionnel! Pourquoi s'effaroucher à Paris de ce qui fait consensus à Bruxelles? Pourquoi s'élever contre l'affirmation, dans une Charte, de valeurs et normes juridiques déjà reconnues par le peuple français par référendum ?

Se laissant emporter par l'action militante de certains de ses membres, les Académies de ce pays ont montré de la science un visage qui, pour tout dire, rend compréhensibles le désamour et la contestation qui se développent vis-à-vis de l'idéologie scientifique dans notre la société. Marquées par un discours scientiste, les prises de position officielles de nos Académies manifestent l'influence, dans les cercles scientifiques, d'intérêts qui ne sont pas ceux de la connaissance. Ces intérêts ne sont pas nécessairement illégitimes, mais il est trompeur de vouloir les travestir en « voix de la science ». Cet épisode témoigne aussi des tensions très actuelles qui traversent la communauté scientifique et des inquiétudes sur l'évolution des conditions d'exercice des métiers qui demandent la mobilisation de compétences scientifiques : chercheurs, médecins, ingénieurs, experts, soumis à de nouvelles exigences de la part de la société. Pour ne pas être en retard d'une guerre, interrogeons-nous sur la manière dont les institutions académiques prennent en charge ces nouvelles demandes et dont les chercheurs peuvent contribuer utilement au débat public plutôt que de laisser certains d'entre eux chercher, en usant d'intrigues byzantines, à le manipuler. 